\title{
Exploring the Relative Importance of Crossing Number and Crossing Angle
}

\author{
Weidong Huang \\ CSIRO ICT Centre \\ PO Box 76 \\ Epping NSW 1710 Australia \\ tony.huang@csiro.au
}

\author{
Maolin Huang \\ University of Technology, Sydney \\ PO Box 123 \\ Broadway NSW 2007 Australia \\ maolin@it.uts.edu.au
}

\begin{abstract}
Recent research has indicated that human graph reading performance can be affected by the size of crossing angle. Crossing angle is closely related to another aesthetic criterion: number of edge crossings. Although crossing number has been previously identified as the most important aesthetic, its relative impact on performance of human graph reading is unknown, compared to crossing angle. In this paper, we present an exploratory user study investigating the relative importance between crossing number and crossing angle. This study also aims to further examine the effects of crossing number and crossing angle not only on task performance measured as response time and accuracy, but also on cognitive load and visualization efficiency. The experimental results reinforce the previous findings of the effects of the two aesthetics on graph comprehension. The study demonstrates that on average these two closely related aesthetics together explain $33 \%$ of variance in the four usability measures: time, accuracy, mental effort and visualization efficiency, with about $38 \%$ of the explained variance being attributed to the crossing angle.
\end{abstract}

\section{Categories and Subject Descriptors}

H.1.2 [Models and Principles]: User/Machine Systems-Human Factors; H.5.0 [Information Interfaces and Presentation]: User Interfaces-Evaluation/Methodology

\section{General Terms}

Performance

\section{Keywords}

Graph drawing, graph visualization, aesthetic criteria, edge crossing, crossing angle, evaluation

\section{INTRODUCTION}

Aesthetics are often used as main criteria in designing automatic graph drawing algorithms. It is commonly believed that graph drawings that conform to aesthetic criteria are more effective for humans to make sense of the embedded information [3]. Examples of aesthetics include minimum number of crossings,

Permission to make digital or hard copies of all or part of this work for personal or classroom use is granted without fee provided that copies are not made or distributed for profit or commercial advantage and that copies bear this notice and the full citation on the first page. To copy otherwise, to republish, to post on servers or to redistribute to lists, requires prior specific permission and/or a fee.

VINCI'10,September 28-29, 2010, Beijing, China.

Copyright $\odot 2010$ ACM 978-1-4503-0436-8/10/09 ..\$10.00. maximum symmetry, and evenly distributed nodes. Most of the currently-in-use aesthetic criteria are originally proposed based on human intuition or personal judgement of the algorithm designer, not on empirical evidence. However, Purchase has conducted a series of user studies in which some of those criteria had been validated for their relevance to the performance of end users in graph comprehension. For example, in a seminal study of Purchase [23] validating individual aesthetic principles, it was found that minimizing edge crossings and minimizing edge bends are both important to human understanding, while the importance of maximizing symmetry remains inconclusive.

Recently the graph drawing and visualization community has seen a growing interest in proposing aesthetic criteria based on empirical evidence [8, 16, 17, 24]. For example, Ware et al. [25] conducted a study examining relative importance of different aesthetics and it was found that path continuity was also an important factor. In a study investigating what kinds of visual arrangements of nodes were preferred in user-generated graph drawings, Ham and Rogowitz [24] found that users often arranged nodes belonging to the same cluster structure in a convex hull, and the convex hull was represented using the cluster's edges.

Among those aesthetic factors proposed based on empirical evidence, maximizing the size of crossing angles has been shown benefi cial for graph comprehension[10, 15, 17]. In particular, in the controlled experiment of Huang et al. [15], sixteen graphs were used. In each of the graphs, there was one path whose length was between four and seven inclusive. There were also separate edges that were used to cross the path, as shown in Figure 1. The crossing angles ranged from zero (no crossings) to ninety degrees with one particular angle size forming one condition; there were seven conditions in total. The shape of the path for each graph remained unchanged across the seven drawings (conditions). Figure 1 showed four drawing examples of a graph used in four of the seven conditions: ninety-degree, fi fty-degree, ten-degree and no-crossing. The subjects were asked to follow the path and count the number of edges of the path from the starting node to the ending node. It was found that in performing the task, the subjects took more time when crossing angles were smaller.

The experiment of Huang et al. led to the crossing angle criterion that maximizing crossing angles can make a graph drawing more readable. This has generated a number of discussions in theoretic research. For example, Dunne and Shneiderman [7] list crossing angle as a "readability metric". Didimo et al. [4] initiate a study of combinatorial questions related to drawing graphs with right angle crossings, followed by Angelini et al. [1], Di Giacomo et al. [5], 


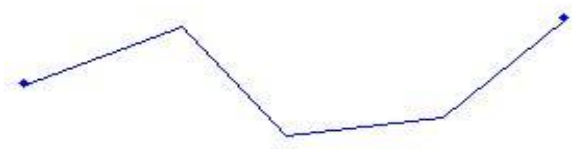

0

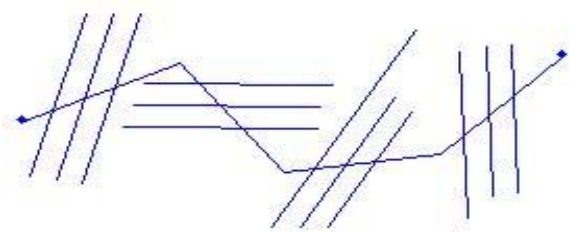

50

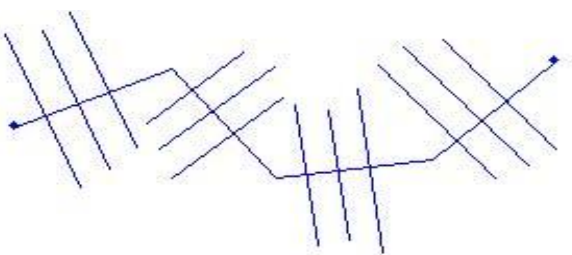

90

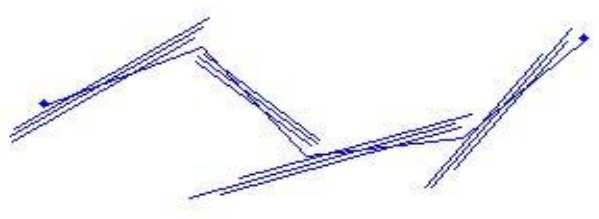

10

\section{Figure1:Drawing examples used in Huangetal.[15](the number below the drawing indicates the size of crossing angles in degrees.0 means no crossings)}

Dujmovic et al. [6], Eades et al. [9, 10], Nguyen et al. [21] and Huang et al. [13]. From a usability point of view, the effect of crossing angle indicates that the same graph can be drawn with a few more crossings, but having the similar level of effectiveness to the corresponding minimum-crossing drawings. This is particular useful given the fact that crossing minimization is a hard problem in graph drawing [11]. In addition, when crossings cannot be completely removed, the negative impact of the remaining crossings can be further reduced by maximizing crossing angles.

However, a closer inspection on the stimuli used in the experiment of Huang et al. revealed that when crossing angle was smaller, the distance between the crossing edges was systematically shorter as well (see Figure 1). The shorter distance between edges made the path harder to follow. Therefore it might also contribute to the longer response time in those smallcrossing-angle drawings. This made the effect of crossing angle less conclusive. Another potential criticism may be that the graphs used in the experiment only had one relative long path and some separate edges, therefore not representative. All this indicates that there is scope for more experimental work to further examine the effect of crossing angle. This is particularly necessary given the potential usefulness of maximizing crossing angles mentioned above. The user study presented in this paper was to address these limitations.

On the other hand, many algorithms take into account more than one aesthetic in their attempt to produce visually pleasing and easy-to-read graph drawings. However, the aesthetics in consideration can be mutually exclusive and compromises have to be made between them. Understanding the relative importance of aesthetic criteria can be useful in deciding which aesthetic should be given priority. In an attempt to obtain a priority order among aesthetics, Purchase [22] conducted an online user study in which fi ve aesthetics were compared for their effects on performance of human graph reading tasks, and it was found that the aesthetic of edge crossings was "by far the most important" factor negatively affecting task performance, followed by the number of bends and display of symmetry. On the other hand, maximizing the minimum angle between neighboring edges and maximizing orthogonality were found having no signifi cant effects. In her study, crossing angle was not part of the investigation, therefore its position on the priority order is unknown. Give that crossing angle is closely related to edge crossings, their relative impact on human graph reading was also considered in the user study presented in this paper.

In graph drawing research, edge crossing has been the most discussed aesthetic in terms of both algorithm design and empirical investigation. It is commonly accepted and employed as a general rule that the number of edge crossings should be minimized whenever possible [3]; many algorithms aim to drawing graphs with minimum or close-to-minimum crossings. Recent empirical research has indicated that the impact of edge crossings varies in different experimental settings. For example, Ware et al. [25] conducted a study in which force-directed graph drawings were used and it was found that in searching the shortest path between two nodes, it was the number of crossings on the shortest path that signifi cantly affected performance, rather than the total number of crossings. In performing simple reasoning tasks, such as "Does this person like to travel to London and Berlin more than to Paris?", with hierarchical graphs of ordered sets, Korner et al. [19] used hand-drawn pictures of a 9-dot and 10-line graph and found that "it is the general disarrangement present in crossed drawings that causes the slower comprehension speed", no matter whether the graph paths in question have crossings or not. Mutzel [20] also mentioned that the collective crossing style can also change the reviewer's perception of the quality of graph drawings. For example, look at the two drawings of the same graph shown in Figure 2. The drawing in Figure 2(a) has 34 crossings, which is $41 \%$ more crossings than the drawing in Figure 2(b) has (24 crossings). However, an informal evaluation revealed that the drawing in Figure 2(a) was perceived having less crossings and being more readable. In our study, force-directed graph drawings were used but the algorithm only involves two basic forces: a spring force between two connected nodes and a repulsive force between all nodes. Given the fi ndings mentioned above, we were also interested to see what effect of edge crossings would be in our study. 


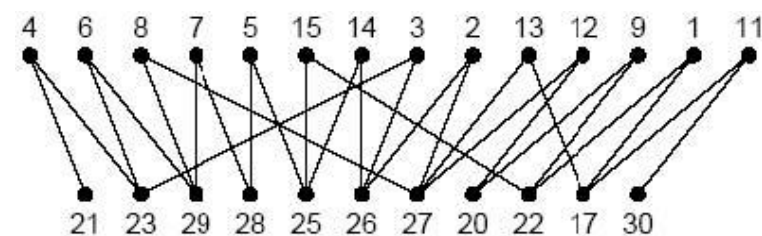

(a)

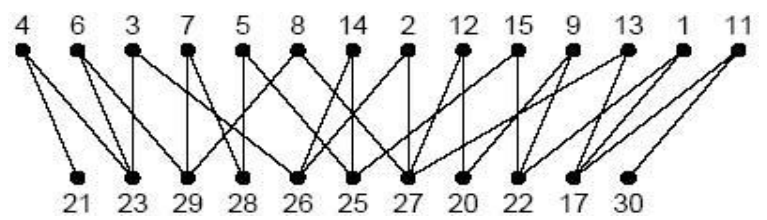

(b)

Figure2:Two drawings of the same graph. (a)k-planarization drawing,(b)minimal-crossing-number drawing. Adopted from Mutzel [20,Figure2].

In addition, previous user studies mainly use performance measures such as task response time and error rate for evaluating graph visualizations. However, performance measures alone have limitations in detecting differences between visualizations and in judging overall quality of visualizations. Huang et al. [12, 14] proposed two additional measures: cognitive load and visualization effi ciency, to address the limitations. In this study, these two measures were used in addition to performance measures.

In summary, the specific purposes of the study are as follows:

1. To replicate the effects of edge crossing and crossing angle on performance of graph comprehension.

2. To further explore the effects of edge crossing and crossing angle on cognitive load and visualization effi ciency.

3. To examine the relative importance of edge crossing and crossing angle.

\section{METHOD}

\subsection{Design}

We first generated a set of drawings using random graphs. The average size of crossing angles and the number of crossings for each drawing were calculated. We then measured task response time, accuracy, cognitive load and visualization effi ciency during the experiment. Based on the data obtained, we examined how these measurements were correlated with size of crossing angle and number of crossings.

Two hundred graphs with size between 10 and 50 were generated and drawn using a spring algorithm, resulting two hundred drawings in total. These drawings were saved beforehand and the average of crossing angles and the number of crossings for each drawing were calculated. Figure 3 shows two drawing examples used in the experiment.

Subjects were asked to fi nd the shortest path between two prespecifi ed nodes in each drawing. The shortest-path search task is considered as a typical task for graph reading, and has been used in various evaluations of graph visualizations (e.g., $[15,25])$. For each trial, the two nodes for each drawing were randomly chosen and highlighted as red with the following conditions:

1.There was only one shortest path between the two nodes. 2.The path length was between 3 and 6 inclusive.

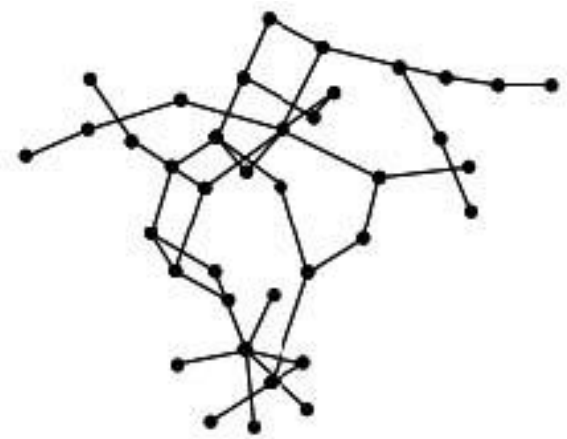

(a)

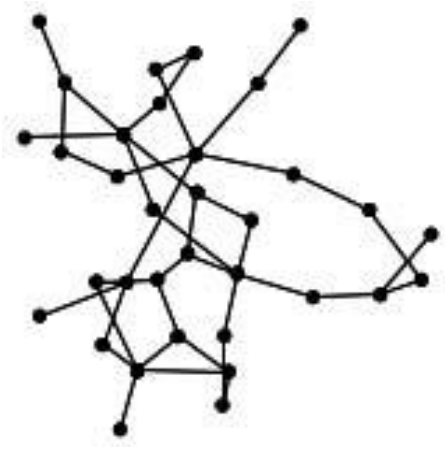

(b)

Figure 3: Drawing examples used in the experiment

During the experiment, the pre-saved drawings were randomly displayed one at a time by a custom-built system and viewed by the subject. For each drawing, the subject's response and task response time were recorded in real time. After the task for each drawing, the subject was also asked to indicate mental effort devoted. The mental effort was rated based on a 9-point scale ranging from 1: "very very low effort" to 9:"very very high effort".

Visualization effi ciency for each drawing was computed using the formula of Huang et al. [12, 14]. Visualization effi ciency is used to measure the overall quality of a visualization by combining individual measures into one formula. It helps to gain insights about cognitive gain (response accuracy) relative to cognitive cost (response time and mental effort) devoted for performing the shortest-path task. First, the individual measures are standardized into z-scores: A for accuracy, $\mathrm{T}$ for time and $\mathrm{R}$ for effort. Then visualization effi ciency $(\mathrm{E})$ is calculated using the formula:

$$
E=\frac{A-T-R}{\sqrt{3}}
$$

In Formula (1), high visualization effi ciency is achieved when high performance accuracy is attained in association with a short response time and low mental effort, and vice versa. If $E=0$, then cognitive gain and cognitive cost are balanced.

Therefore in this experiment, we explored the effects of crossing number and crossing angle on response time, accuracy, mental ef- 
fort and visualization effi ciency. We hypothesized that the size of and metal effort, and positively correlated with accuracy and visualization efficiency. And vice versa for crossing number. Based on prior research of Purchase [22], we also expected that crossing number had a larger effect on each of the four measurements compared to crossing angle.

\subsection{Procedure}

Thirty-two university students with normal or corrected-to-normal vision participated in the experiment. The experiment was conducted in a quiet computer laboratory room. The computers used for all subjects had the same hardware and software specifi cations. First, subjects were given a set of experimental documents to get familiar with graph concepts and node-link diagrams, the online system, the task and the procedure. Then the subjects were asked to practice the system, ask questions and sign the consent form.

During the introduction session, the subjects were told to perform tasks as accurately and as quickly as they could, and to use their eyes only to fi nd the target path without using a mouse to help.

When ready, the subjects indicated to the experimenter and started to perform tasks online. During the experiment, there were two compulsory two-minute breaks that took place at the time when half and three quarters of the drawings had been viewed respectively. The system displayed a message indicating the break, and could only proceed after two minutes had passed. The whole experiment took about 80 minutes on average.

\section{RESULTS}

Our independent variables in this experiment included size of crossing angles measured in degrees (AngleSize) and number of crossings (CrossNumber). The dependant variables included response time (in seconds), accuracy, mental effort and visualization effi ciency. For each drawing, accuracy was computed as a portion of correct trials, while each of the other dependant variables was computed as an average over all subjects. Table 1 shows the mean and standard deviation values of all measured variables.

Table 1: Mean and standard deviation (SD) values

\begin{tabular}{l|c|c}
\hline & Mean & SD \\
\hline \hline CrossNumber & 25.64 & 14.79 \\
AngleSize & 69.36 & 5.00 \\
Time & 14.83 & 5.93 \\
Accuracy & 0.88 & 0.10 \\
Effort & 3.67 & 0.55 \\
Efficiency & -0.93 & 0.53 \\
\hline
\end{tabular}

To test how each of the dependant variables was correlated to the independent variables, we ran simple bivariate regression tests fi rst. The results were shown in Table 2. Figure 4 shows the scatter graphs of dependant variables vs. size of crossing angles, while Figure 5 shows the scatter graphs of dependant variables vs. number of edge crossings.

As can be seen from Table 2 and Figures 4 and 5, both size of crossing angles and number of edge crossings negatively affected task performance. In particular, AngleSize was negatively correlated with response time with correlation $r=-0.43(\mathrm{p}<0.001)$, and positively correlated with accuracy with correlation $\mathrm{r}=0.31$ crossing angle would be negatively correlated with response time ( $\mathrm{p}<0.001)$. In other words, when crossing angles were smaller, the subjects spent more time and made more errors. In contrast with Angle-Size, CrossNumber was positively correlated with response time with $\mathrm{r}=0.59 \quad(\mathrm{p}<0.001)$, and negatively correlated with accuracy with $r=-0.34$ ( $p<0.001)$. In other words, when number of crossings decreased, the subjects spent less time and made fewer errors.

Table 2: Correlations between variables

\begin{tabular}{l|c|c}
\hline & CrossNumber & AngleSize \\
\hline \hline CrossNnumber & & -0.64 \\
AngleSize & -0.64 & \\
Time & 0.59 & -0.43 \\
Accuracy & -0.34 & 0.31 \\
Effort & 0.64 & -0.52 \\
Efficiency & -0.60 & 0.45 \\
\hline
\end{tabular}

Note: All the correlations are significant at the level of $\mathrm{p}<0.001$.

In regard to cognitive load and visualization ef $\mathrm{fi}$ ciency, AngleSize was negatively correlated with mental effort the subjects devoted with $\mathrm{r}=-0.52(\mathrm{p}<0.001)$, and positively correlated with visualization effi ciency with $\mathrm{r}=0.45$ ( $\mathrm{p}<0.001)$. In other words, when crossing angles were smaller, the quality of visualization decreased and more mental effort was needed. In contrast with AngleSize, Cross-Number was positively correlated with mental effort with $\mathrm{r}=0.64 \quad(\mathrm{p}<0.001)$, and negatively correlated with visualization effi ciency with $r=-0.60(p<0.001)$. In other words, when number of crossings decreased, the quality of visualization improved and less effort was needed.

To test the relative importance between crossing number and crossing angle, we ran multiple regression tests by regressing each of the dependant variables on CrossNumber and AngleSize. The results were shown in Table 3 .

As can be seen from Table 3, the overall regression test of response time was statistically signi fi cant, $F(2,197)=54.18$, $\mathrm{p}<0.001$. The two independent variables, CrossNumber and AngleSize, together accounted for $38 \%$ of the variance in time ( $R$ square $=0.38$ ). However, in this regression, only CrossNumber was important, $\mathrm{t}=7.11 \mathrm{p}<0.001$, while AngleSize was not, $\mathrm{t}=-1.30$, $\mathrm{p}>0.05$. The unstandardized coeffi cient (b) for CrossNumber was 0.21 , meaning that for each additional edge crossing, the subject took 0.21 second more time to complete the task, controlling for the size of crossing angles. The unstandardized coeffi cient for AngleSize was 0.11 , suggesting that for one degree increase in crossing angle, 0.11 second less time was needed, controlling for the number of crossings. The relative importance of CrossNumber and AngleSize can be seen from the values of standardized coeffi cients $(\beta)$. Itcanbe seen that each standard deviation (SD) increase in CrossNumber led to a 0.52 SD increase in time, with AngleSize being controlled. On the other hand, each SD increase in AngleSize only resulted in a $0.1 \mathrm{SD}$ decrease in time, controlling for CrossNumber. In other words, CrossNumber had a larger effect than AngleSize did on response time (0.52 vs. 0.10).

Similarly, the overall regression test of accuracy was statistically signifi cant, $\mathrm{F}(2,197)=15.23$, $\mathrm{p}<0.001$. CrossNumber and Angle- 
Size together accounted for $14 \%$ of the variance in accuracy. Each of the two variables had a signifi cant effect on accuracy, with $t=-$ 2.71, $\mathrm{p}<0.01$ for CrossNumber and $\mathrm{t}=1.96, \mathrm{p}<0.05$ for AngleSize. The effect of CrossNumber was slightly larger than that of AngleSize, with $\beta=-0.23$ for CrossNumber and $\beta=0.17$ for AngleSize.

The overall regression test of mental effort was statistically signi fi cant, $F(2,197)=74.64$, p<0.001. CrossNumber and AngleSize
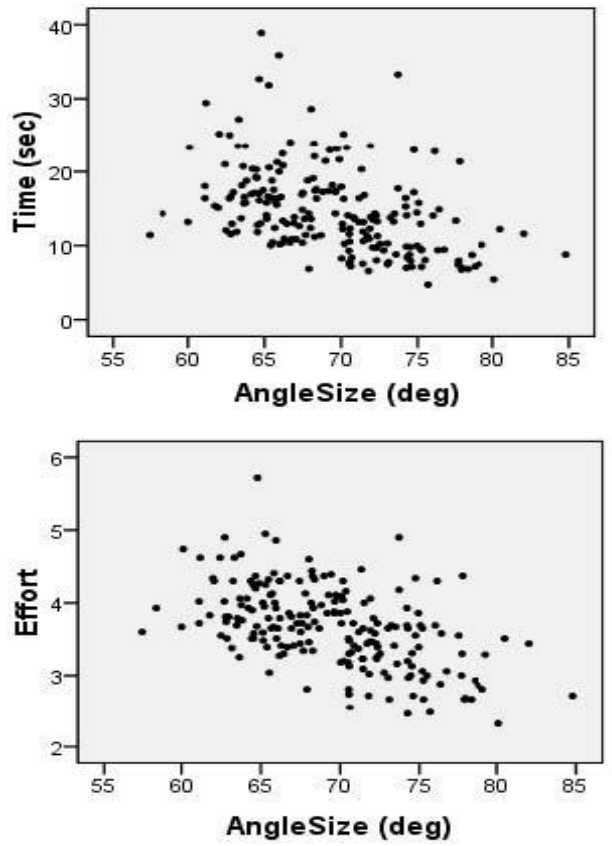

together explained $43 \%$ of the variance in effort. Each of the two variables had a signifi cant effect on effort, with $t=7.46, p<0.001$ for CrossNumber and $t=-2.67, p<0.01$ for AngleSize. The effect of CrossNumber was larger than that of AngleSize, with $\beta=0.52$ for CrossNumber and $\beta=-0.19$ for AngleSize.
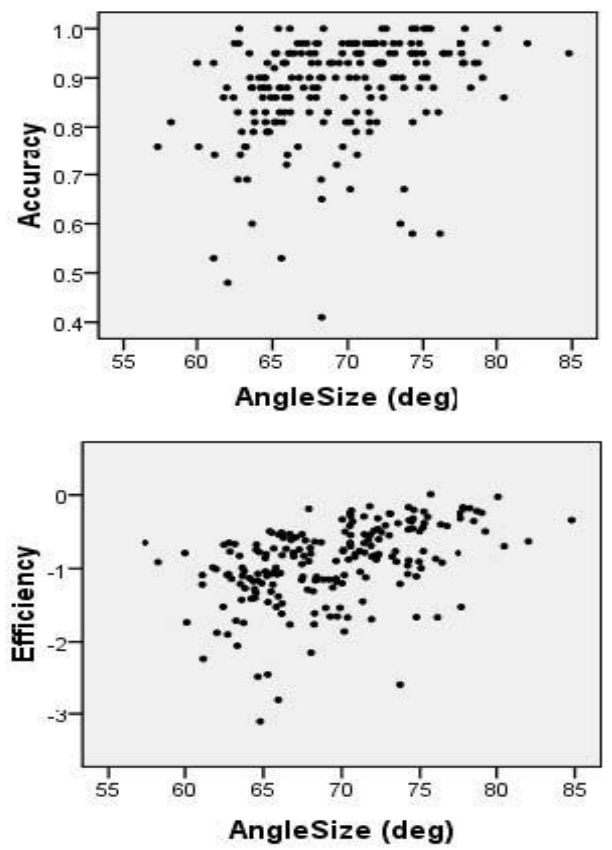

Figure 4: Scatter graphs of dependant variables vs. AngleSize 

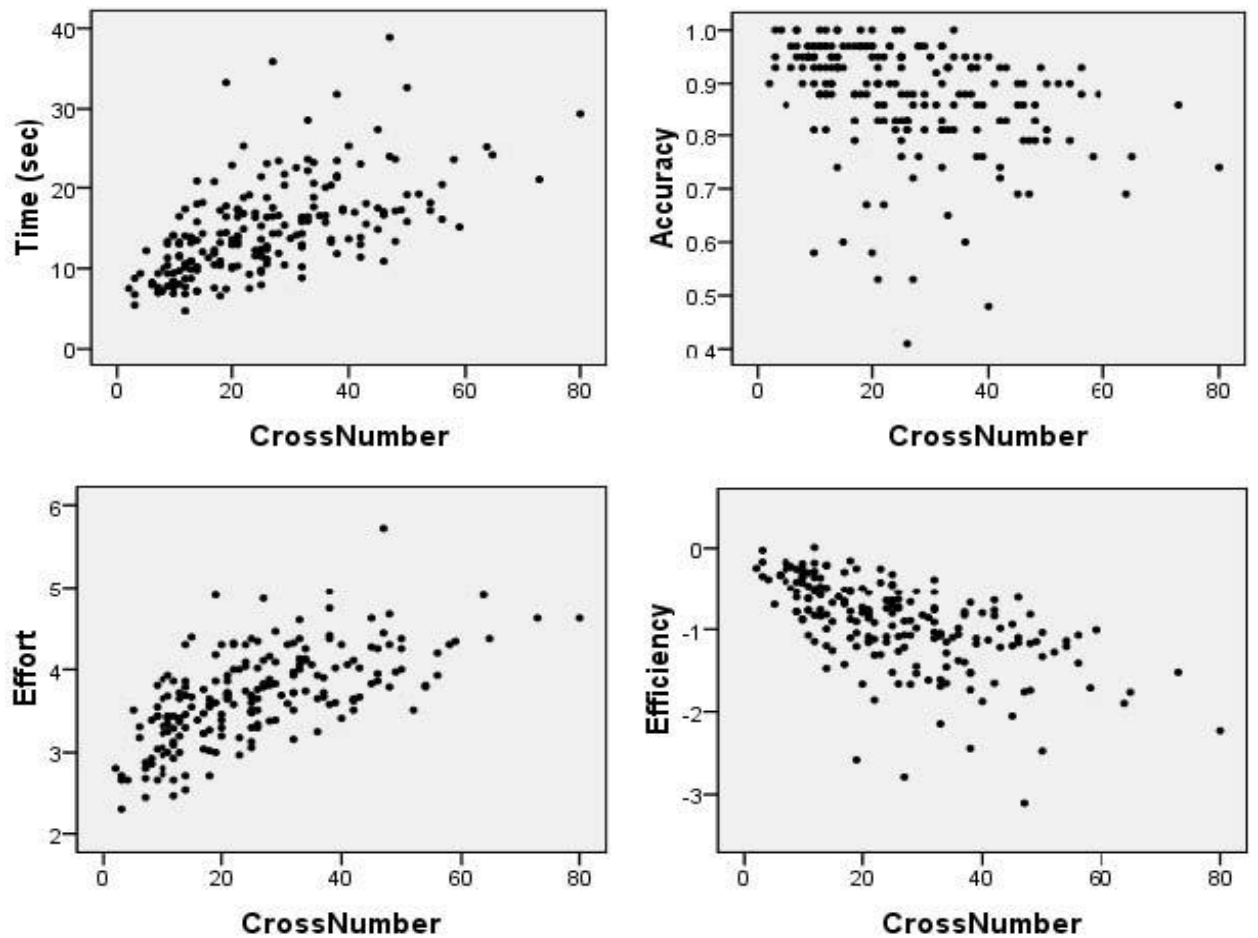

Figure 5: Scatter graphs of dependant variables vs. CrossNumber

Table 3: Coeffi cients and signifi cance of multiple regression tests

\begin{tabular}{|c|c|c|c|c|c|c|}
\hline $\begin{array}{l}\text { Dependant } \\
\text { variables }\end{array}$ & R Square & $\mathrm{F}(2,197)$ & $\begin{array}{l}\text { Independent } \\
\text { variables }\end{array}$ & Unstandardized Coefficients $(b)$ & $\begin{array}{l}\text { Standardized } \\
\text { coefficients }(\beta)\end{array}$ & $\mathrm{t}$ \\
\hline Time & 0.36 & $54.18 * * *$ & $\begin{array}{l}\text { CrossNumber } \\
\text { AngleSize }\end{array}$ & $\begin{array}{c}0.21 \\
-0.11\end{array}$ & $\begin{array}{l}0.52 \\
-0.10\end{array}$ & $\begin{array}{c}7.11^{* * * *} \\
-1.30\end{array}$ \\
\hline Accuracy & 0.14 & $15.23 * * *$ & $\begin{array}{l}\text { CrossNumber } \\
\text { AngleSize }\end{array}$ & $\begin{array}{l}-0.01 \\
0.01\end{array}$ & $\begin{array}{l}-0.23 \\
0.17\end{array}$ & $\begin{array}{c}-2.71^{* *} * \\
1.96 *\end{array}$ \\
\hline Effort & 0.43 & $74.64 * * *$ & $\begin{array}{l}\text { CrossNumber } \\
\text { AngleSize }\end{array}$ & $\begin{array}{c}0.02 \\
-0.02\end{array}$ & $\begin{array}{l}0.52 \\
-0.19\end{array}$ & $\begin{array}{l}7.46^{* * * *} \\
-2.67 * * *\end{array}$ \\
\hline Efficiency & 0.37 & $57.51^{* * * *}$ & $\begin{array}{l}\text { CrossNumber } \\
\text { AngleSize }\end{array}$ & $\begin{array}{l}-0.02 \\
0.01\end{array}$ & $\begin{array}{l}-0.53 \\
0.11\end{array}$ & $\begin{array}{c}-7.24 * * * * \\
1.46\end{array}$ \\
\hline
\end{tabular}

Notes: $*$ denotes $\mathrm{p}<=0.05, * *$ denotes $\mathrm{p}<=0.01$, and $* * *$ denotes $\mathrm{p}<=0.001$.

The overall regression test of visualization efficiency was statistically significant, $F(2,197)=57.51, p<0.001$. CrossNumber and AngleSize together explained $37 \%$ of the variance in effi ciency. However, in this regression, only CrossNumber was important, $\mathrm{t}=-7.24, \mathrm{p}<0.001$, while AngleSize was not, $\mathrm{t}=1.46, \mathrm{p}>0.05$. The effect of CrossNumber was larger than that of AngleSize, with $\beta$ $=-0.53$ for CrossNumber and $\beta=0.11$ for AngleSize.

In summary, the statistical results showed that on average CrossNumber and AngleSize together explained 33\% of variance in the four usability measures: time, accuracy, mental effort and visualization effi ciency, with about $38 \%$ of the explained variance being attributed to AngleSize.

\section{DISCUSSION}

In controlled experiments of graph visualizations, the same graph is often used to produce different conditions by manipulating the layout of nodes (e.g., [14]). Since components of a graph (nodes and edges) are inherently interconnected, manipulating one aesthetic will inevitably change another. This makes the requirement of changing one variable while holding all others constant diffi cult to fulfi 1 . In this experiment, we employed a different approach by using automatically drawn pictures without deliberate manipulation. This allowed us to conduct the graph reading experiment in a more realistic environment. The similar approach was also used by Ware et al. [23].

In examining the effects of aesthetics, task performance measures such as time and error have been used as main criteria. However, performance measures have limitations in detecting differences between visualizations and in giving insight on overall quality of the visualization in consideration. This study extended the examination of crossing number and crossing angle effects to include cognitive load and visualization effi ciency measures as well. 
Our hypotheses on the effects of crossing number and crossing angle were confi rmed in this study. It was found that the size of crossing angle was negatively correlated with response time and mental effort, and positively correlated with accuracy and visualization effi ciency. And vice versa for edge crossings. In regard to the relative importance, it was found that crossing number was more important compared to crossing angle. To be more specifi c, the number of crossings explained about $24 \%$ more of the variance in the dependant variables than crossing angle did. Purchase [20] compared fi ve aesthetic criteria and found that edge crossings had the greatest impact. Our study extended Purchase's priority order to include the aesthetic of crossing angle and the results reinforced the fi nding that edge crossings are the most important.

It should be noted that crossing angles in our drawings were relatively large in general; the averages ranged roughly from 60 to 80 degrees. This might be due to the fact that spring algorithms often produce relatively well laid-out drawings [2]. According to Huang et al. [14], 60 to 80 degrees is the range in which the effect of crossing angle is smallest. If the drawings used in our study had smaller crossing angles, the effect of crossing angle might have become more prominent.

In a study of Ware et al. [23], the total number of edge crossings was found not signifi cantly related to performance of the shortest path tracing in terms of task response time. However, in our study, number of crossings was strongly related to not only task performance, but also cognitive load and visualization effi ciency (see Tables 2 and 3 and Figure 5). Although both studies used the same shortest-path search task, there were a few differences in their experimental settings. For example, Ware et al. used graphs with the same size of 42 , while our study used graphs with size ranging from 15 to 50. A number of aesthetic criteria were considered at the same time by Ware et al., while we only considered crossing angle and crossing number. It is not clear how the effect of total crossing number changed between these two studies.

It is worth mentioning that in testing correlations, we used simple bivariate regressions. Only looking at those linear correlations might not be sufficient for accurate interpretation. However, the significant correlations found in this study were well backed up by corresponding scatterplots shown in Figures 4 and 5. In performing multiple regressions, we had been careful to restrict our attention only to test the relative importance and total effects of the two aesthetics by looking at standardized coefficients and $\mathrm{R}$ squares [17]. Testing a thorough regression model with all possible aesthetics included was beyond the scope of this paper.

It was interesting to see from Table 2 and Figure 6 that the size of crossing angles and the number of crossings were strongly correlated. Considering that the stimuli drawings were generated by a force-directed algorithm, this signifi cant negative correlation was hard to interpret in terms of which depends which. It seems that among the drawings used in this particular study, there was a general pattern that in drawings that had larger crossing angles, the number of crossings was also smaller.

Based on our fi ndings in this study, a practical graph visualization guideline could be that the number of edge crossings should be minimized whenever possible. In cases when crossings cannot be removed, edges should be crossed at an angle as large as possible to reduce the negative impact of the remaining crossings to the minimum.

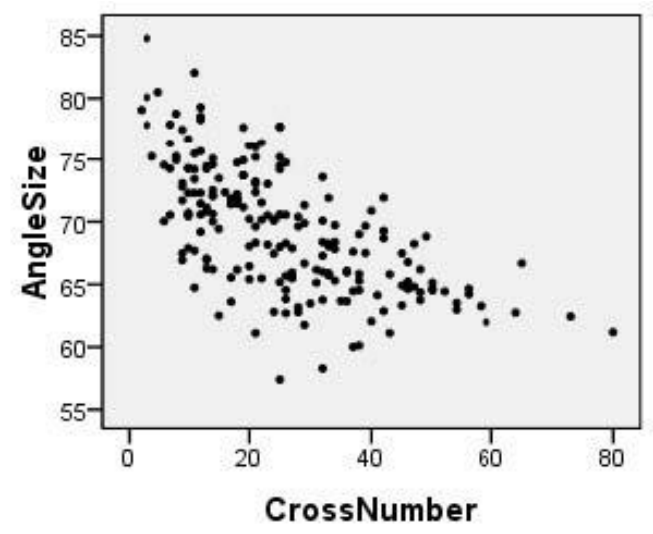

Figure 6: Scatter graph of AngleSize vs. CrossNumber

\section{5. . CONCLUSION}

In this paper we have presented an exploratory study examining the relative impact of crossing number and crossing angle on human performance of the shortest-path search task. In this study, we measured not only task response time and accuracy, but also cognitive load and visualization effi ciency. Multiple regression tests were used for data analysis. The results showed that in general crossing number had a larger effect than crossing angle in each of the four usability measures. However, in our study the experimental stimuli were automatically generated graph drawings in which the crossing angles were in a narrow range of between 60 to 80 degrees.

For future research, we plan to use hand-drawn pictures to further investigate the relative importance between crossing number and crossing angle. By drawing graphs randomly by hand, we are able to make sure that more representative crossing angles are used. We will measure not only the average of crossing angles for the whole drawing, but also the size of crossing angles on the path.

\section{REFERENCES}

[1] Angelini,P., Cittadini, L., Di Battista,G., Didimo, W., Frati, F., Kaufmann, M. and Symvonis, A. (2009) On the Perspectives Opened by Right Angle Crossing Drawings. In Proc. 17th International Symposium on Graph Drawing(GD’09), LNCS 5849, 21-32.

[2] Brandes, U. (2001) Drawing on Physical Analogies. In Michael Kaufmann and Dorothea Wagner (Eds.): Drawing Graphs: Methods and Models. LNCS Tutorial 2025, 71-86.

[3] Di Battista, G., Eades, P., Tamassia, R. and Tollis, I. (1998) Graph Drawing: Algorithms for the Visualization of Graphs, Prentice Hall.

[4] Didimo, W., Eades, P. and Liotta, G. (2009) Drawing Graphs with Right Angle Crossings. In Proc. International Symposium on Algorithms and Data Structures, 206-217.

[5] Di Giacomo, E., Didimo, W., Liotta, G. and Meijer, H. (2009) Area, Curve Complexity, and Crossing Resolution of Non- 
planar Graph Drawings. In Proc. 17th International Symposium on Graph Drawing(GD’09), LNCS 5849, 15-20.

[6] Dujmovic, V., Gudmundsson, J., Morin, P. and Wolle, T. (2010) Notes on Large Angle Crossing Graphs. In Proc.

Computing: the Australasian Theory Symposium.

[7] Dunne, C. and Shneiderman, B. (2009) Improving graph drawing readability by incorporating readability metrics: a software tool for network analysts. Technical Report HCIL2009-13, University of Maryland.

[8] Dwyer, T., Lee, B., Fisher, D., Quinn, K.I., Isenberg, P., Robertson, G. and North, C. (2009) A Comparison of UserGenerated and Automatic Graph Layouts. IEEE Transactions on Visualization and Computer Graphics, 15(6): 961-968.

[9] Eades, P., Hong, S.-H. and Poon, S.-H. (2009) On Rectilinear Drawing of Graphs. In Proc. 17th International Symposium on Graph Drawing(GD’09), LNCS 5849, 232243.

[10] Eades, P., Huang W. and Hong, S.-H. (2009) A ForceDirected Method for Large Crossing Angle Graph Drawing. Technical Report Number 640, University of Sydney.

[11] Garey, M.R. and Johnson, D.S. (1983) Crossing Number is NP-Complete. SIAM Journal on Algebraic and Discrete Methods, 4: 312-316.

[12] Huang, W., Eades, P. and Hong, S.-H (2009) Measuring Effectiveness of Graph Visualizations: A Cognitive Load Approach. Information Visualization, 8(3):139-152.

[13] Huang, W., Hong, S.-H. and Eades, P. (2006) Predicting graph reading performance: a cognitive approach. In Proc. Asia-Pacifi c Symposium on information Visualisation (APVIS'06), 207-216.

[14] Huang, W., Hong, S.-H. and Eades, P. (2008) Effects of Crossing Angles. In Proc. the IEEE Pacifi c Visualization Symposium 2008(Pacifi cVis'08), 41-46.
[15] Huang, W., Hong, S.-H. and Eades, P. (2005) Layout Effects on Sociogram Perception. In Proc. 13th International Symposium on Graph Drawing(GD’05), LNCS 3843, 262273.

[16] Huang, W. (2007) Using Eye Tracking to Investigate Graph Layout Effects. In Proc. Asia Pacifi c Symposium on Visualization (APVIS'07), 97-100.

[17] Keith, T. (2005) Multiple Regression and Beyond. Allyn \& Bacon.

[18] Korner, C. and Albert, D. (2002) Speed of Comprehension of Visualized Ordered Sets. Journal of Experimental Psychology: Applied, 8: 57-71.

[19] Mutzel, P. (1997) An Alternative Method to Crossing Minimization on Hierarchical Graphs. In Proc. the international Symposium on Graph Drawing (GD'96), LNCS 1190, Springer-Verlag, 318-333.

[20] Purchase, H. (1997) Which Aesthetic has the Greatest Effect on Human Understanding? In Proc. the 5th international Symposium on Graph Drawing (GD'97), LNCS 1353, 248 261.

[21] Purchase, H., Cohen, R. and James, M. (1996) Validating Graph Drawing Aesthetics. In Proc. the Symposium on Graph Drawing (GD’05), LNCS 1027, Springer-Verlag, 435446.

[22] Van Ham, F. and Rogowitz, B. (2008) Perceptual Organization in User-Generated Graph Layouts. IEEE Transactions on Visualization and Computer Graphics, 13331339.

[23] Ware, C., Purchase, H., Colpoys, L. and McGill, M. (2002) Cognitive Measurements of Graph Aesthetics. Information Visualization, 1(2): 103-110. 


\section{VINCI 2010 \\ The $3^{\text {rd }}$ Visual Information Communication - International Symposium}

Location: Institute of Software Chinese Academy of Sciences, Beijing China Date: 28-29 September 2010

Conference Chair: Professor Guozhong Dai, Kang Zhang, Maolin Huang

Program Chair: Professor Hongan Wang, Xiaoru Yuan, Linmi Tao, Wei Chen

Sponsors: China Computer Federation

ACM SIGCHI CHINA

Institute of Software, Chinese Academy of Sciences 
The Association for Computing Machinery

2 Penn Plaza, Suite 701

New York New York 10121-0701

ACM COPYRIGHT NOTICE. Copyright $\odot 2010$ by the Association for Computing Machinery, Inc. Permission to make digital or hard copies of part or all of this work for personal or classroom use is granted without fee provided that copies are not made or distributed for profit or commercial advantage and that copies bear this notice and the full citation on the first page. Copyrights for components of this work owned by others than ACM must be honored. Abstracting with credit is permitted. To copy otherwise, torepublish, to post on servers, or to redistribute to lists, requires prior specific permission and/or a fee. Request permissions from Publications Dept., ACM, Inc., fax +1 (212) 869-0481, or permissions@acm . org.

For other copying of articles that carry a code at the bottom of the first or last page, copying is permitted provided that the per-copy fee indicated in the code is paid through the Copyright Clearance Center, 222 Rosewood Drive, Danvers, MA 01923, +1-978-750-8400, +1-978-750-4470 (fax).

Notice to Past Authors of ACM-Published Articles

ACM intends to create a complete electronic archive of all articles and/or other material previously published by ACM. If you have written a work that was previously published by ACM in any journal or conference proceedings prior to 1978, or any SIG Newsletter at any time, and you do NOT want this work to appear in the ACM Digital Library, please inform permissions@acm.org, stating the title of the work, the author(s), and where and when published.

ACM ISBN: 978-1-4503-0436-8 


\section{Preface}

It is our great pleasure to welcome you to Beijing for VINCI 2010, the 2010 Visual Information Communication - International Symposium. VINCI 2010 provides an international forum for researchers and industrial practitioners to discuss the state of the art in visual communication theories, designs, and applications. It brings together researchers and practitioners from many areas, including information visualization, human-computer interaction, $\mathrm{CSCW}$, etc.

The call for papers attracted 37 papers from more than 8 countries and regions. The program committee accepted 18 papers after a thorough review process. These papers cover a wide range of topics, including visual analytics, scientific visualization, document and knowledge visualization, parallel coordinates and graph, new concept and framework, etc.

This year's program includes four keynote speakers: James D. Hollan from UCSD, Benjamin B. Bederson from Maryland University, Cecile Paris from CSIRO and Shengfeng Qin from Brunel University. We believe their talks may benefit all symposium audience. Moreover, it's the first time to open a poster session in VINCI, all poster authors have 2 minutes to present their poster at the conference. It is a good chance to get the entire VINCI audience excited about their work.

VINCI 2010 is made possible by many efforts. We would like to express our deepest thanks to the program committee and organizing team for their tireless work in reviewing papers and making everything ready.

May you be joyful in Beijing, and find inspiration at VINCI 2010. Enjoy the conference!

\section{VINCI 2010 General Chairs and Program Chairs}

September 7, 2010 


\section{Table of Contents}

Keynote Abstract

Beyond the Interface: A Distributed Cognition Perspective

James D. Hollan

The Promise of Zoomable User Interfaces.

Benjamin B. Bederson

Browsing a highly connected information space: can natural language processing help?

Cecile Paris

2D and 3D conceptual design sketching: interface, modelling and progress

Shengfeng Qin

Regular Papers

\section{Session A ： Scientific Visualization}

Difference of Inflow and Outflow based 3D Streamline Placement

Shaorong Wang, Yisong Chen, Sheng Li, Guoping Wang

The Research of the Regional Pollutant Concentration Visualization Based on the Particles.

Zhifang Jiang, Miaomiao Li , Xiangxu Meng, Yan Huang, Shenfang Li

Simulating Self-organizing Behaviors of Fish School.

Ye Yuan, Zhongke Wu

\section{Session B : Parallel Coordinates and Graph}

Visualization of BP Neural Network Using Parallel Coordinates.

Jing Zhao

Column-based Cluster and Bar Axis Density in Parallel Coordinates.

Lei Tang, Xueqing Li, Wenjing Qi, Zhifang Jiang

Exploring the Relative Importance of Crossing Number and Crossing Angle

Weidong Huang, Maolin Huang

\section{Session C : Visualizing the Process}

Semantic Comparison of Structured Visual Dataflow Programs.

Philip T. Cox, Anh Dang

A Model Driven Visualization Platform for Workflow.

Jiayao Chen, Xueqing Li, Jingzhao Yang

Process Visualization to Solve the Travelling Salesman Problem. ...

J. Joshua Thomas, Choy Chee Ken 


\section{Session D : New Concept and Framework}

A Developing Framework for Interactive Temporal Data Visualization.

Xiongfei Luo, Dongxing Teng, Wei Liu, Feng Tian, Guozhong Dai, Hongan Wang

Cooperative Concept Map Based on Cognitive Model for Visual Analysis.

Yi Du, Cuixia Ma, Dongxing Teng, Guozhong Dai

Interactive Visualization with User Perspective: A New Concept.

Quang Vinh Nguyen, Simeon Simoff, Maolin Huang

\section{Session E : Document and Knowledge Visualization}

Harvesting discourse strategies for rapid prototyping of tailored information delivery systems

Shijian Lu, Cécile Paris

TagNetLens: Multiscale Visualization of Knowledge Structures in Social Tags. ...

Liang Gou, Shaoke Zhang, Jing Wang, Xiaolong (Luke) Zhang

Visually Searching the Web for Structural Content.

Hirofumi Miyamoto, Masahito Hirakawa

\section{Session F : Visual Analytics}

A Visual Analytics System for Financial Time-Series Data.

Su Te Lei, Kang Zhang

Event-based Approach to Money Laundering Data Analysis and Visualization.

Tat-Man Cheong , Yain-Whar Si.

An Interactive Sensemaking Framework for Mobile Visual Analytics. ...

133

Anna Wu , Xiaolong(Luke) Zhang, Guoray Cai 


\section{VINCI 2010 Organization}

\section{General Chairs}

Guozhong Dai, Institute of Software Chinese Academy of Sciences, Beijing, China Kang Zhang, University of Texas at Dallas, USA

Maolin Huang, University of Technology, Sydney, Australia

\section{Program Chairs}

Hongan Wang, Institute of Software Chinese Academy of Sciences, Beijing, China

Xiaoru Yuan, Peking University, Beijing, China

Linmi Tao, Tsinghua University, China

Wei Chen, Zhejiang University, China

\section{Organisation Chairs}

Dongxing Teng, Institute of Software Chinese Academy of Sciences, Beijing, China

Lei Ren, Beijing University of Aeronautics and Astronautics, China

Jiawan Zhang,Tianjin University,China

Xiaolong Zhang, Pennsylvania State University,USA

\section{Program Committees}

Paolo Bottoni, University of Rome Sapienza, Italy

Robert Biuk-Aghai, IBM China Research Lab, China

Weijia Cai, VisInsight Inc., China

Xiaochun Cao, Tianjin University, China

Kendra Cooper, University of Texas at Dallas, USA

Gennaro Costagliola, University of Salerno, Italy

Phil Cox, Dalhousie University Canada

Jing Dong, University of Texas at Dallas, USA

Camilla Forsell, Linkoping University, Sweden

Jeff Gray, University of Alabama at Birmingham, USA

Masahito Hirakawa, Shimane U., Japan

Lichan Hong, Xerox PARC Research Center,USA

Tony Huang,CSIRO ICT Centre,Australia

Xiaodi Huang, Charles Sturt University, Australia

Jun Kong, North Dakota State University, USA

Wei Lai, Swinburne University of Technology, Australia

Yang Li, Google Research,Palo Alto, USA

Aidong Lu, University of North Carolina, USA

Xiaoxing Ma, Nanjing University, China

Zhenjiang Miao, Beijing Jiaotong University, China 
Quang Vinh Nguyen, University of Western Sydney, Australia

Monique Noirhomme-Fraiture, University of Namur, Belgium

Yoshihiro Okada, Kyushu University, Japan

Francois Poulet, IRISA, France

Emmanuel Pietriga, INRIA, France

Yu Qian, University of Texas Southwestern Medical Center, USA

Wu Quan, University of Sydney, Australia

Huamin Qu, University of Science \& Technology, Hong Kong

Jose Fernando Rodrigues Jr, University of Sao Paulo at Sao Carlos, Brazil

Guanglei Song, eBay Inc., USA

Nenad Stankovic, Xijiao Liverpool University, China

Zhengxing Sun, Nanjing University, China

Shin Takahashi, University of Tsukuba, Japan

Feng Tian,Institute of Software Chinese Academy of Sciences, Beijing, China

Jie Tian, Institute of Automation,Chinese Academy, China

Wencheng Wang, Institute of Software Chinese Academy of Sciences, Beijing, China

John Wang, Griffith University, Australia

Guido Wirtz, Bamberg University, Germany

Jing Yang, University of North Carolina, USA

Yu-Bin Yang, Nanjing University, China

Liang-Feng Zhu, East China Normal University, China

Shendong Zhao,National University of Singapore, Singapore

Xueqing Li, Shangdong University, China

Qingyi Hua, Northwest University, China 